\title{
Topical and Intradermal Efficacy of Photodynamic Therapy with Methylene Blue and Light-Emitting Diode in the Treatment of Cutaneous Leishmaniasis Caused by Leishmania braziliensis
}

\author{
Mônica Raquel Sbeghen¹, Evandra Maria Voltarelli² , Tácito Graminha Campois ${ }^{1}$, Elza Kimura ${ }^{3}$, Sandra \\ Mara Alessi Aristides ${ }^{4}$, Luzmarina Hernandes ${ }^{5}$, Wilker Caetano ${ }^{6}$, Noboru Hioka ${ }^{6}$, Maria Valdrinez \\ Campana Lonardoni ${ }^{4}$, Thaís Gomes Verzignassi Silveira ${ }^{4 *}$ \\ 'Universidade Estadual de Maringá, Maringá, Paraná, Brazil \\ ${ }^{2}$ Center for Zoonosis Control, Maringá, Paraná, Brazil \\ 32Departament of Pharmacy, Universidade Estadual de Maringá, Maringá, Paraná, Brazil \\ ${ }^{4}$ Department of Clinical Analyses and Biomedicine, Universidade Estadual de Maringá, Maringá, Paraná, Brazil \\ ${ }^{5}$ Department of Morphophysiology Sciences, Universidade Estadual de Maringá, Maringá, Paraná, Brazil \\ ${ }^{6}$ Department of Chemistry, Universidade Estadual de Maringá, Maringá, Paraná, Brazil
}

\section{Correspondence to \\ Thaís Gomes Verzignassi \\ Silveira, Department of Clinical \\ Analyses and Biomedicine, \\ State University of Maringá, \\ Maringá, Paraná, Brazil. \\ Tel: +55-4430114878 \\ Email: tgvsilveira@uem.br}

Published online 28 June 2015

\begin{abstract}
Introduction: The topical and intradermal photodynamic therapy (PDT) effect of methylene blue (MB) using light-emitting diode (LED) as light source (MB/LED-PDT) in the treatment of lesions of American cutaneous leishmaniasis (ACL) caused by Leishmania braziliensis in hamsters were investigated.

Methods: Hamsters were infected in the footpad with $4 \times 10^{7}$ promastigotes of L. braziliensis and divided in 4 groups: Control group was not treated, $A m B$ group was treated with amphotericin $B, M B-I d$ group received intradermal $M B$ at the edge of the lesion and MB-Tp group received $M B$ topic. After treatment with MB, the animals were illuminated using red LEDs at the 655 nm wavelength for 1 hour. The MB/LED-PDT was carried out three times a week for 12 weeks. Results: Animals of MB-Tp group presented lesion healing with significant diminution in extent of the lesion, and reduced parasite burden compared to control group; however, no significant difference was seen compared to the AmB group. MB-Tp group also showed reconstitution of the epithelium, the formation of collagen fibers, organization in the epidermis, a little disorganization and inflammation in the dermis. MB-Id was ineffective in all parameters evaluated, and it was comparable to the control group results.

Conclusion: These data show that PDT with the use of MB-Tp and LED may be an alternative for the treatment of ACL. However, additional studies are being conducted to assess the potential of MB/LED-PDT, alone or in combination with conventional therapy, for the treatment of ACL. Keywords: Photodynamic therapy; Cutaneous leishmaniasis; Leishmania braziliensis; Treatment
\end{abstract}

\section{Introduction}

American cutaneous leishmaniasis (ACL) occurs in the Americas from southern United States to northern Argentina. ACL has clinical importance due to the high incidence and severe clinical manifestations. ${ }^{1}$ ACL can have 2 forms, the skin (the most frequent form of the disease with skin lesion) and mucocutaneous (partial or total destruction of mucous membranes surrounding the nasopharynx region). ${ }^{1-3}$ There may also be disseminated disease with various skin ulcers, and the diffuse disease characterized by nodular lesions without the presence of ulceration. ${ }^{2}$
ACL has been treated with pentavalent antimonials (N-methylglucamine), as the preferential drug, and polienics antibiotics (amphotericin B), aminoglicosideos (paromomycin) and antiprotozoal agent (pentamidine isothionate) ${ }^{4}$ but they can lead to serious side effects and discontinuation of treatment by the patients. In recent years, alternative treatments have been used for ACL and among them, photodynamic therapy (PDT) has shown promising results. ${ }^{4,5}$

PDT builds on the application of a photosensitizer compound into the target tissue that is illuminated with light

Please cite this article as follows: Sbeghen MR, Voltarelli EM, Campois TG, et al. Topical and intradermal efficacy of photodynamic therapy with methylene blue and light-emitting diode in the treatment of cutaneous leishmaniasis caused by Leishmania braziliensis. J Lasers Med Sci. 2015;6(3):106-111. doi:10.15171/jlms.2015.03. 
at the appropriate wavelength. After the photosensitizer is excited, it can promote the production of oxygen species (superoxide anion and hydroxyl radical), capable of inducing irreparable oxidative damage to cells ${ }^{6}$ (type I reaction). Additionally, the photosensitizer may lead to the formation of singlet oxygen (type II reaction). PDT has been used in the therapy of tumors (skin, bladder, lung, esophagus), the post-surgical microbiological control for acne, fungal and bacterial infections as well as in ophthalmology and dentistry. ${ }^{6}$

Treatment of leishmaniasis with PDT showed complete lesion healing with the elimination of amastigotes forms in animal models. ${ }^{5,7}$ In humans, good results were also obtained in the treatment of this disease, including esthetic results. ${ }^{7}$ The side effects observed in the treatment with PDT were rare, but the discomfort caused by the heat when lasers were used as light source were reported. ${ }^{9} \mathrm{Hy}-$ perpigmentation and erythema in the treatment site were also reported. ${ }^{9}$

The high costs related to lasers restrict their use as light source in PDT, but the use of light-emitting diode (LED) may be affordable even for low incoming people. Also, the use of some photosensitizer compounds may increase the costs of the treatment.

Methylene blue (MB) is a dye-photosensitizer useful for PDT, which is excited by red light. This compound has advantages such as low cost, easy to purchase, antifungal and antibacterial activity. ${ }^{10,11} \mathrm{MB}$ presents little harm to the patient in the treatment, and its toxic potential can be activated by light. ${ }^{12} \mathrm{MB}$ is also employed as medications for the treatment of methemoglobinemia (injected intravenously) and other diseases. Thus, this study aimed to evaluate the PDT using MB and LED in the treatment of ACL in hamsters infected with Leishmania braziliensis.

\section{Methods}

Chemical and LED Light Source

$\mathrm{MB}$, molecular formula $\mathrm{C}_{16} \mathrm{H}_{18} \mathrm{ClN}_{3} \mathrm{~S} .3 \mathrm{H}_{2} \mathrm{O}$ with molar mass of 373.90 g. $\mathrm{mol}^{-1}$ (Vetec) was prepared in water $0.5 \%$ type I or saline $(0.9 \%)$. The light source was LED (Everlight) system containing 6 units (in a serie) that emit red light, with $663 \mathrm{~nm}$ wavelength, $5 \mathrm{~mW} . \mathrm{cm}^{-2}$ each LED.

\section{Parasite}

Leishmania (Viannia) braziliensis (MHOM/BR/1987/ M11272), were maintained in medium 199 (Gibco, Invitrogen Corporation) containing $10 \%$ fetal calf serum, $2 \mathrm{mM}$ L-glutamine, $1 \%$ sterile human urine, and antibiotics (penicillin G $100 \mathrm{IU} / \mathrm{mL}$ and streptomycin $100 \mu \mathrm{g} / \mathrm{mL}$ ) until exponential growth phase.

\section{Experimental Infection}

Golden hamsters, females, 90 days old, obtained from the Central Animal Facility at the State University of Maringá were used. The animals were inoculated with $4 \times 10^{7}$ promastigotes of L. braziliensis in $100 \mu \mathrm{L}$ of saline $(\mathrm{NaCl}$ $0.9 \%)$ in the right footpad. On the left footpad, $100 \mu \mathrm{L}$ of saline were inoculated as the control. Before the infection, the animals received the anesthetics xylazine $10 \mathrm{mg} / \mathrm{kg}$ (Calmiun Animal Health Agener-Union) and ketamine $50 \mathrm{mg} / \mathrm{kg}$ (Francotar ${ }^{\circledR}$ - Virbac Animal Health). The animals were examined weekly for evaluation of lesion development.

Photodynamic Therapy with the system MB/LED-PDT After all hamsters had developed lesions (approximately 90 days after infection), the animals were separated into 4 groups ( $n=10$ animals each group). Control group: the animals received no treatment. AmB group: the animals were treated intraperitoneally with amphotericin B (5 mg/ $\mathrm{kg}$ /day) for 20 days with a break of 7 days and further injection for 7 days (positive control). MB-Id group: the animals received $50 \mu \mathrm{L}$ of $10 \mathrm{mM} \mathrm{MB}$ in saline, inoculated intradermally at a single point on the outer edge of the lesion. The lesion was illuminated with LED for 1 hour. MB-Tp group: the animals received $50 \mu \mathrm{L}$ of $10 \mathrm{mM} \mathrm{MB}$ in water, applied topically on the lesion. The lesion was illuminated with LED for 1 hour. The animals of MB-Id and MB-Tp groups were treated 3 times a week, during 12 weeks. ${ }^{12}$

\section{Evaluation of the Lesion}

The evaluation of the lesion was performed weekly by measuring the thickness of the footpad (infected and non-infected footpad) with a thickness gauge (Mitutoyo, Japan) and the difference between the measurements were calculated. Photographies of the lesions were taken monthly.

Quantification of L. braziliensis in Lymph Node and Spleen

Quantification of L. braziliensis was performed in the regional lymph node and spleen of hamsters according to Buffet et al. ${ }^{13}$ Briefly, the animals were euthanized, and the spleen and popliteal lymph node of each animal were excised aseptically and weighed. The organs were disrupted in 199 culture medium (Gibco, Invitrogen Corporation, NY, USA) and the suspensions were homogenized several times with a needle $(0.45 \mathrm{~mm} \times 13 \mathrm{~mm})$ to break the cells. A 1:4 dilution of each sample was transferred to a 96-well plate and incubated at $25^{\circ} \mathrm{C}$. The plates were observed under a light microscope inverted once a week for 30 days. The highest dilution that was detected at least one parasite was considered as the titer. The experiments were performed in duplicates. The parasite burden was determined for each organ and was considered as the geometric mean titers multiplied by initial dilution of the suspension organs in the plate (16.7) and divided by the organ weight (in grams).

\section{Histological Evaluation}

The tissue of the lesion area (including the damaged and healthy tissue surrounding the lesion) was removed, fixed with $4 \%$ paraformaldehyde for 24 hours and embedded in paraffin. Semi-serial sections of $5 \mu \mathrm{m}$ thickness were made and placed on slides for staining with hematoxylin 
and eosin for morphological observation and immunohistochemical for detection of fragmented DNA.

The skin morphology was observed microscopically to the occurrence of histopathological alterations (presence of inflammatory infiltrations and structural alterations). These alterations and the presence of amastigote in macrophages were stratified as absent $(-)$, weak $(+)$, moderate $(++)$, or severe $(+++)$ according to the frequency of the cells in the dermis. For each animal, ten histological sections were analyzed in an Olympus BX41 microscope.

"In situ" Detection of Fragmented DNA - TUNEL Assay DNA fragmentation was detected using an apoptosis detection kit (ApopTag ${ }^{\circledR}$ Plus Peroxidase In Situ, Millipore, MA, USA) according to the manufacturer guideline. Harris hematoxylin was used for counterstaining.

\section{Statistical Analysis}

The normality was tested for all results by Shapiro Wilk's, and the data were analyzed by Mann-Whitney $U$ test using the Statistica 7.0 software. The differences between the data were considered at the $5 \%$ level.

\section{Ethical Aspects}

The use of laboratory animals and the experimental trials were approved by the Ethics Committee on the Use of Animals in experimentation at the Universidade Estadual de Maringá (Report 111/2009 on 3/11/2009).

\section{Results}

Evolution of the Lesions

Figure 1 shows the evolution of lesions of the groups of animals throughout the treatment. The animals of Control group followed the evolution of the lesion, and the animals of AmB group showed a reduction and healing

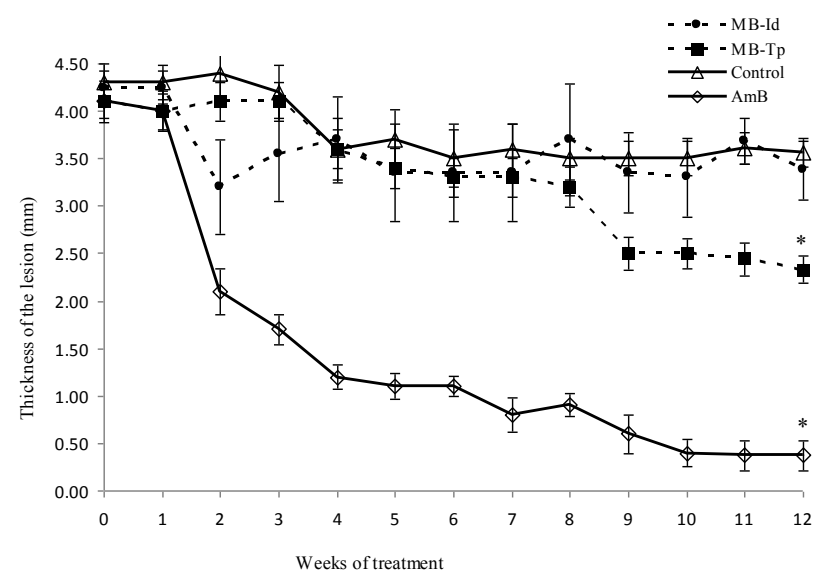

Figure 1. Effect of MB/LED-PDT on lesions of hamsters infected with Leishmania braziliensis. The animals were infected with $4 \times$ $10^{7}$ promastigotes of $L$. braziliensis and the evolution of lesions was evaluated weekly for 12 weeks after initiation of treatment with MB/LED-PDT. Group MB-Id received methylene blue (MB) intradermally, and MB-Tp received MB topically. Group AmB was treated with amphotericin B and Control group was not treated. The values represent mean \pm SEM ( $n=10$ animals). ${ }^{*} P<.05$, difference between the beginning and the end of the treatment. of the lesions. No reduction or healing of the lesions were observed in the animals of MB-Id group compared to Control group, but a significant reduction and healing of the lesion was observed in about $30 \%$ of the animals from MB-Tp group after 9 weeks of treatment. Treatment was initiated 12 weeks after infection when all animals had similar lesions $(P>.05)$. At the end of treatment (22 weeks) the thickness of lesion of the MB-Tp group was significant lower than Control $(P=.0023)$ and MB-Id $(P=.0038)$ groups, but no significant difference was observed between MB-Id and Control groups $(P=.4230)$. The thickness of lesion of the group $\mathrm{AmB}$ was lower than MB-Id $(P=.0007)$ and MB-Tp $(P=.0005)$ groups.

By comparing the thickness of the lesion at the beginning and end of treatment in each group, there was difference in $\mathrm{MB}-\mathrm{Tp}(P=.0006)$ and $\mathrm{AmB}(P=.0007)$ groups, but not for MB-Id $(P=.1562)$ and Control $(P=.0520)$ groups. Figure 2 shows the evolution of the lesions in the footpad of the animals at the beginning and the end of treatment.

Quantification of L. braziliensis in Spleen and Lymph Node

After of treatment the hamsters were euthanized, and parasite burden in spleen and the lymph node were evaluated. In the MB-Tp group of parasitic burden in the lymph node was significantly lower (Figure 3A) than in the MB-Id

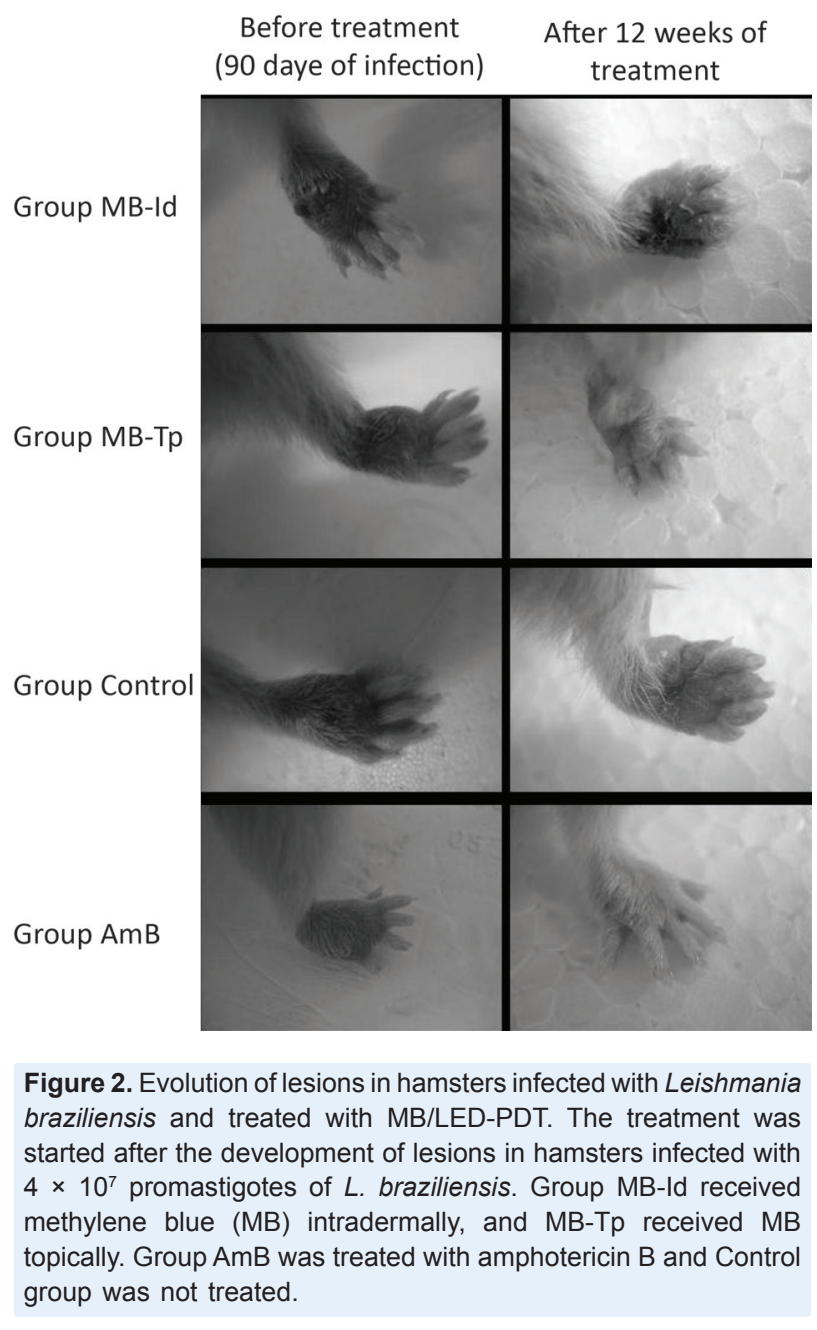


group $(P=.0034)$, but it was not different the $\mathrm{AmB}$ group $(P=.2332)$ and to the Control group $(P=.1451)$.

The parasite burden in the spleen was also significantly lower in the MB-Tp group than in the control group $(P=.0017)$ and MB-Id group $(P=.0123)$, but no difference was found between MB-Id and Control group $(P=.0832)$. The reduction of the parasite burden in the of the parasites in the spleen of the MB-Tp group was similar to the hamsters treated with $\mathrm{AmB}(P=1.0000)$ (Figure 3B).

The lymph nodes of the MB-Tp animals had a significant reduction in their weight compared to Control group $(P=.0012)$ and MB-Id groups $(P=.0020)$, and it was comparable to the AmB group $(P=1.0000)$. The weight of lymph nodes of MB-Id group was comparable to the Control group $(P=.092)$, and it was higher than AmB group $(P=.0032)$.

In the MB-Tp group the spleen of the animals presented lower weight than in the untreated animals (control) $(P=.0379)$, but no difference was seen when it was compared to the MB-Id $(P=.0542)$ or AmB $(P=.3359)$ groups. The weight of the spleen in the MB-Id group exceeded to the AmB group $(P=.0013)$, and it was comparable to the Control group $(P=1.000)$.
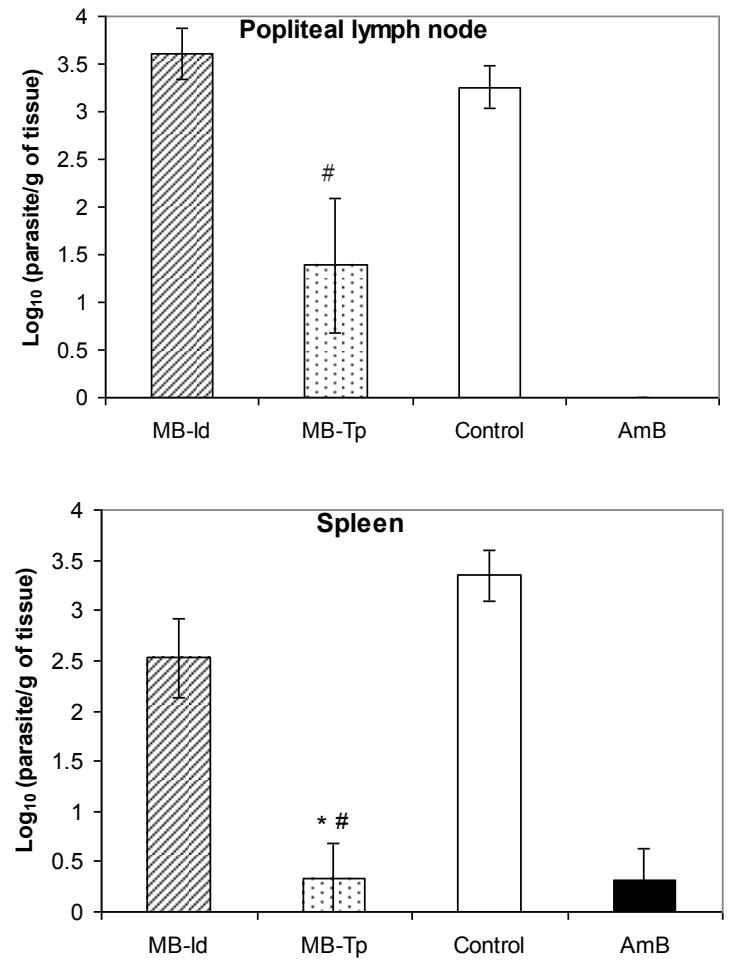

Figure 3. Quantification of $L$. braziliensis in hamsters treated with MB/LED-PDT. $4 \times 10^{7}$ promastigotes of $L$. braziliensis was inoculated in the footpad of hamsters, and the animals were treated with MB/LED-PDT. Group MB-Id received methylene blue (MB) intradermally $(n=8)$, and group MB-Tp received MB topically $(n=9)$. After treatment for 12 weeks, the parasites were quantified in the lymph nodes and spleen. Group AmB was treated with amphotericin $B(n=8)$ and Control group was not treated $(n=9)$. The results are mean \pm SEM. ${ }^{*} P<.05$ compared to the not treated group; \# $P<.05$ compared to PDT MB-Id group.
Histopathological Analysis

The presence of amastigotes of Leishmania in tissue and gross alterations in the lesions was seen in the Control group. Also, epithelialized areas, naked and others in the reepithelialization process were observed. A disorganized dermis region was seen, with the loss of normal morphology, filled by an intense inflammatory infiltrate (rich in polymorphonuclear and mononuclear) and punctuated by the large number of lymph capillaries with the large lumen. In this group the keratinocytes in the epidermis and dermal fibroblasts presented strong staining by TUNEL assay.

MB-Tp treatment significantly recovered the lesion area (Figure $1 \mathrm{~A}$ and $\mathrm{B}$ ). A regenerated epidermis and dermis with bundles of collagen fibers was seen by microscope examination with a significant reduction in the number of lymphatic capillaries. Despite TUNEL staining revealing a higher frequency of labeled cells, a lower staining intensity compared to the Control group was seen. Rare macrophages infected with amastigotes were found in animals treated with PDT (MB-Tp).

A noticeable recovery of the injured tissue with reduced inflammation and dilated lymphatic vessels was observed with treatment with AmB. However, the dermis still had mild inflammatory infiltrate, and less dense bundles of collagen fibers compared to the MB-Tp. TUNEL staining showed a lower frequency of labeled cells and lower intensity staining compared to the MB-Tp (Figure 4).

Animals from MB-Id group were not evaluated because they did not show significant therapeutic effects in the other parameters. The score for inflammation is represented in Table 1.

\section{Discussion}

In this paper, the use of MB/LED-PDT by 2 different administration routes (topical and intradermal) for the treatment of ACL in hamsters experimentally infected with $L$. braziliensis was evaluated. The topical administration of MB/LED (MB-Tp) decreased significantly the lesion and the thickness of the footpad and improved the lesion's healing. There was also a reduction in parasite burden of the spleen and regional popliteal lymph node, as well as a reduction of the weights of these organs (data not shown) when compared to nontreated animals. Also, some of these parameters did not differ from those obtained from animals treated with $\mathrm{AmB}$, showing a similar efficiency of $\mathrm{MB} / \mathrm{LED}$-PDT in the treatment of ACL. However, the intradermal treatment (MB-Id) was not effective to treat ACL. These animals showed similar results to nontreated animals (control) for the parameters evaluated.

Some photosensitizer compounds used in PDT in in vivo experiments undergo self-aggregation and precipitate when in contact with the aqueous body tissues, decreasing the singlet oxygen formation and reducing the effects of PDT. For intradermal inoculation, the MB was dissolved in saline but, in the presence of endogenous compounds may favor the formation of aggregates or inactivate the ability to generate the reactive oxygen. For topical applica- 


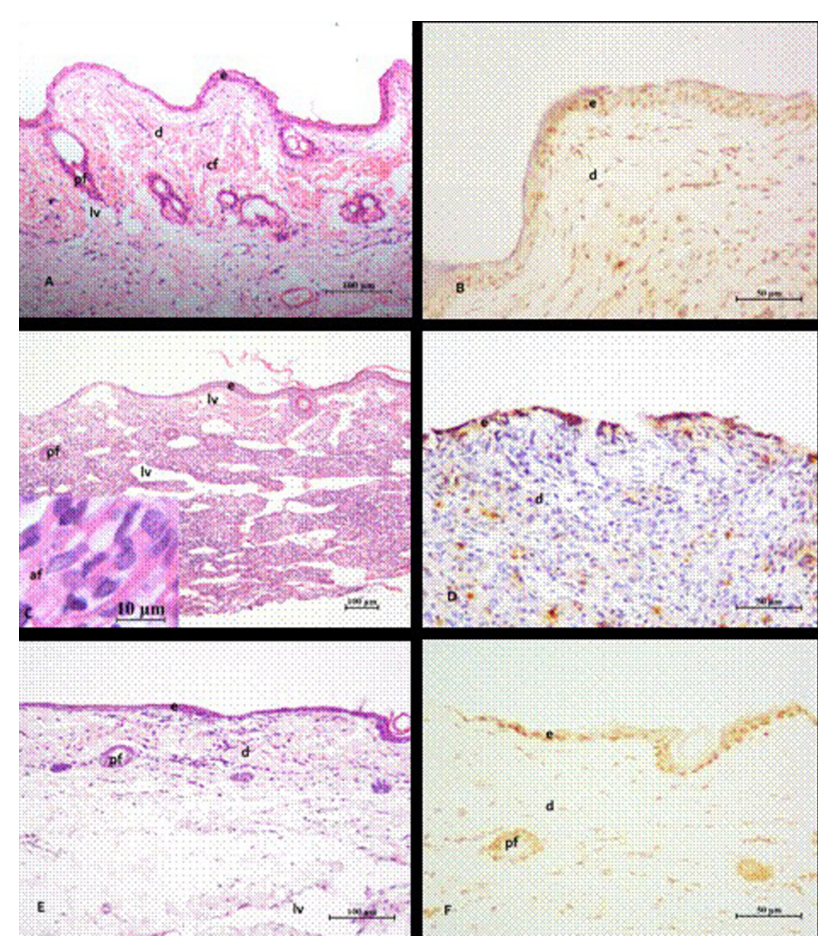

Figure 4. Photomicrograph of the footpad lesions of hamsters infected with Leishmania braziliensis. Lesions treated with MB/ LED-PDT with methylene blue (MB) administered topically ( $T p$ ) $(A$ and $B)$. The control animals $(C$ and $D)$ were untreated or were treated with amphotericin $B(A m B)(E$ and $F)$. After $M B /$ LED-PDT (A), intact skin with hair follicles (pf) in regeneration, absence of inflammation and few dilated lymphatic vessels (Iv) are noted. The dermis (d) showed thick bundles of collagen fibers (cf). In infected untreated animals (C), intense inflammatory infiltrate intermingled with dilated lymphatic vessels are noted. These lesions showed partial loss of epidermis (e) and frequent occurrence of amastigote forms (af) (detail in C) in the dermis region. After treatment with $A m B(E)$, regenerated epidermis and dermis in the repair process, with beams of smaller and thinner collagen fibers are noted. In ( $B, D$ and $F)$ the sections stained with TUNEL showed that higher frequency of labeled cells, particularly fibroblasts and keratinocytes occurred in lesions treated with MB/LED-PDT, followed by those treated with AmB and those not treated. Stain: hematoxylin and eosin (HE) (A, C and E); TUNEL (B, D and F).

tion, the MB was dissolved in water to reduce the chance of MB self-aggregation. This fact could explain the treatment failure of MB-Id treatment. In addition, the trauma caused by the inoculation of $\mathrm{MB}$ on the edge of the lesion may have contributed to the nonhealing process of the lesion and also liquid extravasation with release of proteins which may sequestrate $\mathrm{MB}$ from the site.

As seen in our previous study published elsewhere, ${ }^{12} \mathrm{MB}$ alone has no effect against ACL, but according to the results presented in this study, the topical application of MB and LED was effective. Studies using EtNBSe and LASER, as well as ALA and LASER in murine cutaneous leishmaniasis (CL) by L. major showed reduction in parasite burden of the lesion, in necrosis and in inflammation of the region of lesion. ${ }^{8,14}$

The animals that received MB-Tp showed histological features similar to those found in animals treated with AmB, such as reconstitution of the epithelium, the organization of the dermis, with fibroblasts and collagens fibers, suggesting that MB/LED-PDT contributed to the healing process of the lesion. Rare macrophages infected with amastigotes were found in animals treated with MB-Tp, which were not observed in animals treated with AmB.

Apoptosis is a process that can clear cells without activation of the immune response and it is very important to the pathogenesis of a disease. Leishmania apoptosis can occur in response to antileishmanial drugs. Investigation about the extent of parasite apoptosis and host cell apoptosis that occur in vivo and how these two events contribute to infection can be important to the infection control. ${ }^{15}$ Apoptotic cells were found in higher frequency in the lesions of animals treated with topic MB/LED-PDT than in animals treated with $\mathrm{AmB}$, followed by those nontreated. Akilov et $\mathrm{al}^{16}$ demonstrated the control of murine CL progression, induced by Treg cells, and reduction of the parasite burden after treatment with apoptotic macrophage. Treg cells regulate the balance Th1 and Th2, that is important in the Leishmania infection control. In this context, the presence of apoptotic cells in animals treated with MB-Tp can be related to the disease control.

Akilov et $\mathrm{al}^{8}$ treated murine CL by L. major with EtNBSe and LASER and found after 7 days of treatment that the necrosis were at the center of the lesions and epidermis remained viable, which could not be identified in our study in animals treated with MB-Tp. These same authors, using ALA and LASER, observed the intense inflammatory infiltrate of lymphocytes and histiocytes in the treated tissue, amastigotes of Leishmania donovani inside macrophages and little hyperkeratosis and hyperplasia in the epidermis, coinciding with the histological features found in the group treated with MB-Tp.

Gardlo et $\mathrm{al}^{5}$ treated a human patient with CL and observed the presence of amastigotes of $L$. donovani and infiltrates of lymphocytes and decreased levels of antibodies against $L$. donovani. Enk et $\mathrm{al}^{7}$ treated patients with CL caused by L. major, with ALA applied in solution with water and oil before the LASER (wavelength in the red area) and demonstrated a reduction in lesion size, elimination

Table 1. Score Representing the Reconstitution of the Epithelium, Inflammation of the Epidermis and Dermis and the Presence of Amastigotes in Macrophages, of the Hamsters Infected With Leishmania braziliensis, Treated or not Treated

\begin{tabular}{lllll}
\hline Groups & Reconstituition of the Epithelium & Inflammation in the Epidermis & Inflammation in the Dermis & Amastigotes in Macrophages \\
\hline $\begin{array}{l}\text { Control } \\
\text { (untreated) }\end{array}$ & - & +++ & +++ & ++ \\
MB-Tp & ++ & + & + & + \\
AmB & +++ & - & - & - \\
\hline
\end{tabular}

$(-)$ absence, $(+)$ weak, $(++)$ moderate, $(+++)$ severe. 
of amastigotes in smears of the lesion, complete healing of the lesion and a little hyperpigmentation at the site.

Several studies with PDT used LASER as a light source ${ }^{7,14,17}$ and several compounds as photosensitizer substances. ${ }^{8,17-19}$ Although the LED unit is less potent than LASER, the price of LED is much lower. Furthermore, the array of the LED devices presents radiation per area higher than the LASER and the red LED emits light in the range of 655 $\mathrm{nm}$ wavelength, which penetrates deeper into the tissue and is relevant to the success of PDT. Moreover, this wavelength coincides with the region of maximum absorption of the MB. ${ }^{20,21}$

In conclusion, our results show that PDT with $\mathrm{MB}$ as a photosensitizer substance and LED as the light source, improved the healing of lesions, reconstituted the epithelial tissue organization in the dermis and epidermis, and reduced the parasite burden of popliteal lymph node and spleen, in hamsters experimentally infected with L. braziliensis. These data suggest the use of MB/LED-PDT may be an alternative for the treatment of ACL. However, additional studies are being conducted to assess the potential of $\mathrm{MB} / \mathrm{LED}-\mathrm{PDT}$, alone or in combination with conventional therapy for the ACL treatment.

\section{Conflict of Interest}

All authors report to not have any commercial relationship or funding and conflicts of interest related to this study.

\section{Acknowledgments}

The work received financial support of the Fundação Arau-cária and Coordenação de Aperfeiçoamento de Pessoal de Nível Superior (Capes).

\section{References}

1. Gontijo B, Carvalho MLR. Leishmaniose tegumentar Americana. Rev Soc Bras Med Trop. 2003;36:71-80. doi:10.1590/s0037-86822003000100011.

2. Reis AB, Gontijo CM. Leishmaniose Tegumentar Americana. In: Neves DP, ed. Parasitologia Humana. 12th ed. São Paulo, SP: Editora Ateneu; 2011:49-65.

3. World Health Organization. Leishmaniasis. http://www. who.int/topics/leishmaniasis/en/. Accessed Jul 17, 2014.

4. Brasil. Ministério da Saúde. Secretaria de Vigilância em Saúde. 2013. Manual de Vigilância da Leishmaniose Tegumentar Americana, 2nd ed, Editora do Ministério da Saúde, Brasília, DF. http://portal.saude.gov.br/portal/ arquivos/pdf/manual2_lta_2ed.pdf

5. Gardlo K, Horska Z, Enk CD, et al. Treatment of cutaneous leishmaniasis by photodynamic therapy. J Am Acad Dermatol. 2003;48:893-896. doi:10.1067/ mjd.2003.218.

6. Machado AE. Terapia Fotodinâmica: princípios, potencial de aplicação e perspectivas. Química Nova. 2000;23:237-243.

7. Enk C, Fritsch C, Jonas F, et al. Treatment of cutaneous leishmaniasis by photodynamic therapy. Arch Dermatol. 2003;139:432-434. doi:10.1001/archderm.139.4.432.

8. Akilov OE, Kosaka K, O’Riordan K, Hasan T. Parasiticidal effect of $\mathrm{d}$-aminolevulinic acid-based photodynamic therapy for cutaneous leishmaniasis is indirect and mediated through the killing of the host cells. Exp Dermatol. 2007;6:1067-1075. doi:10.1111/ j.1600-0625.2007.00578.x.

9. van der Snoek EM, Robinson DJ, van Hellemond JJ, Neumann HA. A review of photodynamic therapy in cutaneous leishmaniasis. J Eur Acad Dermatol Venereol. 2008;22:918-922. doi:10.1111/j.14683083.2008.02805.x.

10. Wainwright M, Crossley K B. Methylene blue a therapeutic dye for all seasons. J Chemother. 2002;14:431-443. doi:10.1179/joc.2002.14.5.431.

11. Calzavara-Pinton PG, Venturini M, Sala R. A comprehensive overview of photodynamic therapy in the treatment of superficial fungal infections of the skin. J Photochem Photobiol B Biol. 2005;78:1-6. doi:10.1016/j. jphotobiol.2004.06.006.

12. Peloi LS, Biondo CE, Kimura E, et al. Photodynamic therapy for American Cutaneous Leishmaniasis: the efficacy of methylene blue in hamsters experimentally infected with Leishmania (Leishmania) amazonensis. Exp Parasitol. 2011;12:353-356.

13. Buffet PA, Sulahian A, Ganin YJF, Nassar N, Deuroin F. Culture microtitration: a sensitive method for quantifying Leishmania infantum in tissues of infected mice. Antimicrob Agents Chemother. 1995;39:21672164. doi:10.1128/aac.39.9.2167

14. Akilov OE, Kosaka K, O’Riordan K, Hasan T. Photodynamic therapy for cutaneous leishmaniasis: the effectiveness of topical phenothiaziniums in parasite eradication and Th1 immune response stimulation. Photochem Photobiol Sci. 2007;6:10671075. doi:10.1039/b703521g.

15. Shaha C. Apoptosis in Leishmania species \& its relevance to disease pathogenesis. Indian J Med Res. 2006;123:233-244.

16. Akilov $\mathrm{OE}, \mathrm{Wu} \mathrm{MX}$, et al. Vaccination with photodynamic therapy-treated macrophages induces highly suppressive T-regulatory cells. Photodermatol Photoimmunol Photomed. 2011;27:97-107.

17. Akilov OE, Kosaka K, O'Riordan $\mathrm{K}$, et al. The role of photosensitizer molecular charge and structure on the efficacy of photodynamic therapy against Leishmania parasites. J Chem Biol. 2006;13:839-847.

18. Bristow CA, Hudson R, Paget TA, Boyle RW. Potential of cationic porphyrins for photodynamic treatment of cutaneous Leishmaniasis. Photodiagnosis Photodyn Ther. 2006;3:162-167.

19. Dutta S, Ray D, Kolli BK, Chang KP. Photodynamic sensitization of Leishmania amazonensis in both extracellular and intracellular stages with aluminum phthalocyanine chloride for photolysis in vitro. Antimicrob Agents Chemother. 2005;49:4474-4484. doi:10.1128/aac.49.11.4474-4484.2005

20. Sternberg ED, Dolphin D. Pyrrolic photosensitizers. Curr Med Chem. 1996;3:293-324.

21. Sternberg ED, Dolphin D, Bruckner C. Porphyrin-based photosensitizers for use in photodynamic therapy. Tetrahedron. 1998;54:4151-4202. doi:10.1016/S00404020(98 )00015-5. 